Pesq. Vet. Bras. 30(5):447-452, maio 2010

\title{
Intoxicação por alcaloides pirrolizidínicos em ruminantes e equinos no Brasil ${ }^{1}$
}

\author{
Ricardo B. Lucena², Daniel R. Rissi², Lisanka A. Maia ${ }^{3}$, Mariana M. Flores ${ }^{4}$, \\ Antônio Flávio M. Dantas ${ }^{5}$, Verônica M. da T. Nobre ${ }^{5}$, Franklin Riet-Correa ${ }^{5}$ \\ e Claudio S.L. Barros ${ }^{6 *}$
}

\begin{abstract}
Lucena R.B., Rissi D.R., Maia L.A., Dantas A.F.M., Flores M.A., Nobre V.M.T., Riet-Correa F. \& Barros C.S.L. 2010. [Poisoning by pyrrolizidine alkaloids in ruminants and horses in Brazil.] Intoxicação por alcaloides pirrolizidínicos em ruminantes e equinos no Brasil. Pesquisa Veterinária Brasileira 30(5):447-452. Laboratório de Patologia Veterinária, Departamento de Patologia, Universidade Federal de Santa Maria, Av. Roraima 1000, Cidade Universitária, Camobi, Santa Maria, RS 97105-900, Brazil. Email: claudioslbarros@uol.com.br

Cases of poisoning by pyrrolizidine alkaloids (PAs) in ruminants and horses were surveilled retrospectively by accessing the files of two veterinary diagnostic laboratories in southern and northeastern Brazil. The data obtained were compared with those withdrawn from the literature and pertaining to outbreaks of the toxicosis in Brazil where it is associated with the ingestion of PAs-containing plants from the genera Senecio, Crotalaria and Echium. Acute and chronic forms of the toxicosis were encountered. Acute disease was observed in association with the ingestion of Crotalaria retusa in sheep and goats. $C$. retusa and Senecio spp. were also responsible for chronic poisoning in cattle, horses and sheep. PAs poisoning is an important cause of death in livestock in Brazil. It is the major cause of death in cattle in the Central region of Rio Grande do Sul and one of the major causes of death in horses in the state of Paraíba. The epidemiology, clinical signs, pathology, and importance of acute and chronic toxicoses are described and discussed.
\end{abstract}

INDEX TERMS: Diseases of cattle, sheep, goat and horses, poisonous plants, pyrrolizidine alkaloids, Senecio spp., Crotalaria spp., plant poisoning.

RESUMO.- Casos de intoxicação por alcaloides pirrolizidínicos (APs) em ruminantes e equinos foram investigados retrospectivamente através do acesso aos arquivos de dois

\footnotetext{
${ }^{1}$ Recebido em 30 de outubro de 2009.

Aceito para publicação em 5 de janeiro de 2010.

2 Programa de Pós-Graduação em Medicina Veterinária, área de concentração em Patologia Veterinária, Laboratório de Patologia Veterinária, Centro de Ciências Rurais (CCR), Universidade Federal de Santa Maria (UFSM), Av. Roraima 1000, Cidade Universitária, Camobi, Santa Maria, RS 97105-900, Brasil.

3 Graduação em Medicina Veterinária, Hospital Veterinário, Centro de Saúde e Tecnologia Rural (CSTR), Universidade Federal de Campina Grande (UFCG), Campus de Patos, Rodovia Patos-Teixeira Km 1, Av. Universitária, Santa Cecília, Patos, PB 58700-970, Brasil.

${ }^{4}$ Graduação em Medicina Veterinária, Laboratório de Patologia Veterinária, CCR, UFSM, Santa Maria, RS.

${ }^{5}$ Hospital Veterinário, CSTR, UFCG, Campus de Patos, Santa Cecília, Patos, PB.

${ }^{6}$ Laboratório de Patologia Veterinária, Departamento de Patologia, UFSM, Cidade Universitária, Camobi, Santa Maria, RS. "Autor para correspondência: claudioslbarros@uol.com.br
}

laboratórios de diagnóstico veterinário no Sul e Nordeste brasileiro. Os dados obtidos foram comparados com aqueles retirados da literatura concernentes a surtos dessa toxicose no Brasil, onde ela é associada com a ingestão de plantas que contêm APs dos gêneros Senecio, Crotalaria e Echium. Formas aguda e crônica da toxicose foram encontradas. A doença aguda foi observada em associação com a ingestão de Crotalaria retusa em ovinos e caprinos. $C$. retusa e Senecio spp. também foram responsáveis pela intoxicação crônica em bovinos, equinos e ovinos. A intoxicação por APs é uma importante causa de morte em animais pecuários no Brasil. Essa é a principal causa de morte em bovinos na região Central do Rio Grande do Sul e uma das principais causas de morte em equinos na Paraíba. A epidemiologia, os sinais clínicos, a patologia e a importância da intoxicação por APs são descritos e discutidos.

TERMOS DE INDEXAÇÃO: Doenças dos bovinos, ovinos, caprinos e equinos, plantas tóxicas, alcalóides pirrolizidínicos, Senecio spp., Crotalaria spp., intoxicação por planta. 


\section{INTRODUÇÃO}

Alcaloides pirrolizidínicos (APs) são fitoquímicos de ocorrência natural em cerca de 6.000 espécies de plantas de diversos gêneros e famílias (Culvenor 1980). São compostos químicos estáveis e são bioativados no fígado pelas enzimas monooxigenases de função mista para metabólicos tóxicos denominados ésteres pirrólicos (deidropirrolizidinas) e alcoóis pirrólicos (McLean 1970, Prakash et al. 1999). Os derivados pirrólicos são agentes alquilantes altamente reativos que inibem a mitose, causando megalocitose e morte celular (Prakash et al. 1999). Os gêneros Senecio, Crotalaria, Heliotropium e Echium abrigam as principais espécies de plantas envolvidas na intoxicação espontânea por APs em herbívoros e humanos (Cheeke 1998).

No Brasil são descritas as formas aguda e crônica da intoxicação por APs em herbívoros. As plantas envolvidas nesses casos pertencem aos gêneros Senecio, Crotalaria e Echium. Os objetivos do presente trabalho são descrever a epidemiologia, os achados clínico-patológicos e os aspectos comparativos da intoxicação por APs em ruminantes e eqüinos. Para isso foi realizado um estudo retrospectivo de surtos de intoxicação por APs diagnosticados no Laboratório de Patologia Veterinária (LPV) da Universidade Federal de Santa Maria (UFSM), Rio Grande do Sul, e no Laboratório de Patologia Animal (LPA) da Universidade Federal de Campina Grande (UFCG), Paraíba.

\section{MATERIAL E MÉTODOS}

Foram revisados os protocolos de necropsia com diagnóstico de intoxicação por alcaloides pirrolizidínicos (APs) em bovinos, equinos, ovinos e caprinos, constantes dos arquivos do LPV/ UFSM e do LPA/UFCG. Dos protocolos foram colhidas informações referentes à epidemiologia, aos sinais clínicos, aos achados de necropsia e à histopatologia. Foi feita uma revisão sistemática das publicações de casos de intoxicação espontânea por APs de ocorrência espontânea no Brasil para que se pudesse comparar os achados descritos na literatura com os achados deste estudo retrospectivo.

\section{RESULTADOS}

\section{Intoxicação aguda por alcalóides pirrolizidínicos (APs) em ruminantes}

Surtos da intoxicação aguda por APs foram observados em ovinos e caprinos no Nordeste brasileiro. Dois surtos da intoxicação aguda por APs em ovinos associados à ingestão de Crotalaria retusa foram diagnosticados no LPA/ UFCG. O primeiro surto ocorreu no estado da Paraíba nos meses de agosto e setembro. De um rebanho de 80 ovinos morreram 16 (20\%) animais de diferentes idades, após uma evolução clínica de 12 horas. O segundo surto foi diagnosticado em agosto no estado do Rio Grande do Norte. Em um rebanho de 150 ovinos morreram 34 (22,6\%). A evolução clínica da doença foi de 2-5 dias. Os sinais clínicos em ambos os surtos incluíam anorexia, apatia, icterícia, ascite e incoordenação. Na necropsia observaram-se hemorragias na serosa de múltiplos órgãos, fígado com acentuação do padrão lobular, hidropericárdio, hidrotórax, ascite, distensão da vesícula biliar e icterícia. Histologicamente, as lesões hepáticas caracterizavam-se por necrose centrolobular difusa.

Em caprinos foi observado um surto da intoxicação aguda por APs associado à ingestão de $C$. retusa no município de Patos, Paraíba. De um lote de 30 caprinos adoeceram e morreram quatro (13,33\%). A susceptibilidade dos caprinos à intoxicação por $C$. retusa foi confirmada experimentalmente pela administração da planta (doses tóxicas de $5 \mathrm{~g} / \mathrm{kg}, 6 \mathrm{~g} / \mathrm{kg}$ e $10 \mathrm{~g} / \mathrm{kg}$ ). Os sinais clínicos, achados de necropsia e histopatologia nos casos da intoxicação natural e experimental por APs em caprinos foram semelhantes aos descritos anteriormente para essa toxicose em ovinos. Diferenças no quadro clínico em caprinos incluem cólicas, além de bruxismo e o não desenvolvimento de icterícia.

\section{Intoxicação crônica por APs em bovinos}

De 1969 até 2008 haviam sido diagnosticados 484 casos de intoxicação por Senecio spp. em bovinos no LPV/ UFSM. Isso equivale a $23 \%$ de todas as causas de morte confirmadas em bovinos necropsiados nesse laboratório. A idade dos bovinos afetados variou de dois meses a 15 anos. A evolução do curso clínico foi de 2-30 dias e os sinais clínicos mais frequentes incluíam apatia, diminuição do apetite, isolamento do rebanho, pelos arrepiados, tenesmo, prolapso de reto, emagrecimento progressivo, diarreia, incoordenação, ascite e sinais neurológicos como agressividade, andar compulsivo e pressão da cabeça contra objetos. Numa pequena proporção de casos foram observadas icterícia e opistótono. Fotossensibilização e cegueira foram raramente observadas. Os achados de necropsia mais frequentes incluíam fígado acentuada e difusamente firme e com cápsula cinzenta. Em muitos dos casos havia nódulos de regeneração de vários tamanhos no parênquima hepático, os nódulos ás vezes eram vermelhos ou amarelo-alaranjados (degeneração gordurosa). Outras lesões frequentes foram edema das dobras do abomaso, edema do mesentério, distensão da vesícula biliar, com edema da parede e às vezes com hiperplasia polipóide, ascite, hidrotórax, edema dos linfonodos mesentéricos e hemorragias nas serosas da cavidade abdominal. Histologicamente havia hepatomegalocitose, fibrose dissecante ou difusa, com perda de hepatócitos e proliferação de ductos biliares. Menos frequentemente observaram-se nódulos de regeneração e bilestase. Na vesícula biliar havia hiperplasia adenomatosa da mucosa. No encéfalo havia degeneração esponjosa (status spongiosus) da substância branca.

No LPA/UFCG foram diagnosticados três surtos de intoxicação crônica por Crotalaria retusa em bovinos. O primeiro surto ocorreu no município de Patos e foram afetados dois bovinos. O segundo surto foi observado no município de São José do Bonfim e um bovino foi afetado de um total de seis. No município de Soledade foi diagnosticado o terceiro surto. De um total de 20 bovinos, cinco 
adoeceram e dois morreram. A duração dos sinais clínicos até amorte ou eutanásia dos bovinos variou de 48 horas até 90 dias. Todos os bovinos manifestaram fotossensibilização, perda de peso, incoordenação e decúbito. $\mathrm{Na}$ necropsia, o fígado era firme e em algumas áreas o padrão lobular estava acentuado. Havia edema da parede e hiperplasia polipóide na mucosa da vesícula biliar. Histologicamente havia hepatomegalocitose e degeneração multifocal de hepatócitos, fibrose periportal, proliferação de ductos biliares, pericolangite e hiperplasia adenomatosa da mucosa da vesícula biliar.

\section{Intoxicação crônica por APs em equinos}

No LPV/UFSM foi realizada uma única necropsia de um equino intoxicado por Senecio spp., proveniente de um surto da intoxicação que afetou cinco equinos no município de Fortaleza dos Valos. Os equinos tiveram sinais de diminuição progressiva do apetite, fotossensibilização, movimentos constantes da cabeça e pressão da cabeça contra objetos. A morte do equino necropsiado ocorreu 15 dias após o início dos sinais clínicos. Na necropsia o fígado deste equino tinha a superfície irregular e com acentuação do padrão lobular. Adicionalmente havia hemorragia na mucosa do cólon e do ceco. Histologicamente havia hepatomegalocitose, necrose individual de hepatócitos, fibrose, formação de nódulos de regeneração hepatocelular, proliferação de ductos biliares e bilestase canalicular. Astrócitos Alzheimer tipo II, isolados ou em grupos, ocorriam no córtex telencefálico e nos núcleos da base.

A intoxicação crônica por APs em equinos se mostrou muito mais importante no Nordeste brasileiro. De 1993 a 2008 foram diagnosticados 36 casos de intoxicação crônica por $C$. retusa em equinos no LPA/UFCG. Esses equinos eram provenientes dos estados da Paraíba e do Ceará, correspondendo a aproximadamente $16 \%$ das necropsias em equinos. Os sinais clínicos eram característicos de encefalopatia hepática e incluíam depressão, andar a esmo, pressão da cabeça contra objetos e galopes sem rumo. Menos frequentemente foi observada fotossensibilização. Os achados de necropsia e histopatológicos foram semeIhantes aos descritos acima para a intoxicação crônica por Senecio sp. no equino do LPV/UFSM. Diferenças incluíam edema pulmonar em alguns casos.

\section{Intoxicação crônica por APs em ovinos}

A intoxicação crônica por plantas contendo APs em ovinos foi observada em três surtos e um caso isolado no Rio Grande do Sul e em um surto na Paraíba. O primeiro surto observado no Rio Grande do Sul ocorreu no município de São Gabriel em um campo altamente infestado por Senecio brasiliensis. Foram realizadas seis necropsia em ovinos (não foi informado a morbidade, mortalidade e o tempo de evolução dos sinais clínicos nesse surto). O segundo surto observado no Rio Grande do Sul ocorreu em Mata, numa área também infestada por $S$. brasiliensis. Havia 94 ovinos nessa área, dos quais $51(54,25 \%)$ adoeceram e 50 (53,20\%) morreram. Nove ovinos doentes foram mantidos em obser- vação até a morte ou eutanásia (30 dias a um ano). Outro surto ocorreu no município de São Vicente do Sul, em área infestada por Senecio spp. Nessa ocasião, morreram sete ovinos, dos quais um foi necropsiado (não foi informado o número total do rebanho); a letalidade foi de $100 \%$. Por fim, um caso isolado foi observado no município de Santa Maria em uma ovelha adulta. Todos os ovinos intoxicados por Senecio spp. demonstraram fotossensibilização, apatia, anorexia e emagrecimento progressivo. Adicionalmente, seis ovinos tiveram icterícia e hemoglobinúria; em quatro desses animais foram confirmados níveis elevados de cobre no fígado e rim (llha et al. 2001). Alguns ovinos afetados pressionavam a cabeça contra objetos e dois demonstraram agressividade. Na necropsia, o fígado de todos os ovinos estava diminuído de volume, firme, difusamente marrom-amarelado e com nodulações aleatórias na superfície natural e de corte. Nos seis ovinos com quadro de hemoglobinúria, o rim estava tumefeito, friável e marrom-escuro. Histologicamente havia fibrose hepática, principalmente nas regiões periportais, mas que se estendia entre os lóbulos formando fibrose em ponte. Outras alterações histológicas incluíam proliferação de ductos biliares, bilestase, hepatomegalocitose, degeneração e nódulos de regeneração. No rim dos ovinos com hemoglobinúria havia vacuolização e necrose das células epiteliais dos túbulos contorcidos proximais, gotas hialinas no citoplasma das células tubulares e cilindros na luz tubular. No encéfalo de todos os ovinos foi observada degeneração esponjosa (status spongiosus).

No município de São José do Bonfim, Paraíba, foi observado um surto de intoxicação crônica por APs em ovinos que haviam estado em um campo invadido por $C$. retusa 40 dias antes do início da doença. De um total de 10 ovinos, sete $(70 \%)$ adoeceram e morreram após um curso clínico de 24-48 horas. Os ovinos afetados manifestaram apatia, anorexia, diarreia, pressão da cabeça contra objetos e decúbito lateral. Na necropsia, o fígado estava firme ao corte e havia acentuação do padrão lobular e distensão da vesícula biliar. Havia ainda ascite, edema do abomaso e do mesentério e derivações portossistêmicas. Histologicamente havia fibrose hepática periportal, proliferação de ductos biliares, hepatomegalocitose e bilestase. Na substância branca do encéfalo foi observado status spongiosus. Não foram observados sinais clínicos ou lesões decorrentes de hemoglobinúria.

\section{DISCUSSÃO}

O diagnóstico de intoxicação por plantas contendo APs nos casos revisados foi baseado na epidemiologia, nos sinais clínicos, nos achados de necropsia e histopatológicos. Parte desses dados havia sido relatada anteriormente pelas equipes do LPV/UFSM e do LPA/UFCG e podem ser consultados para obtenção de informações mais detaIhadas (Barros et al. 1987, 1992, Dantas et al. 2004, Gava \& Barros 1997, llha et al. 2001, Nobre et al. 1998, Nobre et al. 2004a,b, 2005, Pilati \& Barros 2007, Pimentel et al. 2009, Rissi et al. 2007). 
Os resultados deste trabalho demonstram a importância da intoxicação por APs como causa de morte em ruminantes e equinos no Brasil, corroborando dados apresentados em publicações anteriores. No Brasil, a intoxicação aguda e/ou crônica em ruminantes e equinos por APs são causadas pela ingestão de plantas dos gêneros Senecio (Asteraceae) e Crotalaria (Leguminosae) e, menos fequentemente, pela ingestão de Echium plantagineum (Boraginaceae). A intoxicação espontânea na forma crônica por Senecio sp. foi descrita em bovinos (Barros et al. 1987, 1992, Mendéz et al. 1987), equinos (Carvalho \& Maugé 1946, Curial \& Guimarães 1958, Gava \& Barros 1997), ovinos (llha et al. 2001) e búfalos (Corrêa et al. 2008). As espécies de Senecio envolvidas nesses surtos incluem S. brasiliensis, S. oxyphyllus, S. heterotrichius, S. selloi, S. cisplatinus, S. tweedieie S. madagascariensis (Méndez et al. 1987, Méndez \& Riet-Correa 1990, Barros et al. 1992, Cruz et al. 2010). A intoxicação natural por $C$. retusa foi descrita na forma crônica em equinos (Nobre et al. 2004) e ovinos (Dantas et al. 1999) e na forma aguda em ovinos (Nobre et al. 2005). Intoxicação crônica por $C$. juncea foi observada em equinos (Nobre et al. 1994) e a intoxicação crônica por uma espécie não identificada de Crotalaria foi descrita em bovinos (Lemos \& Barros 1998). Adicionalmente há um relato da intoxicação crônica por $E$. plantagineum em bovinos (Méndez et al. 1985).

Estudos demonstram variação no teor de APs nas diferentes espécies de plantas ou em diferentes partes de uma mesma planta. Quando analisadas todas as partes de $C$. retusa (folhas, flores, frutos e raiz), a concentração do alcaloide monocrotalina variou de $0,16 \%$ a $0,5 \%$ (Nobre et al., 2005). Por outro lado, quando analisadas apenas as sementes, a concentração de monocrotalina em $C$. retusa foi de até $6,84 \%$ (Anjos et al. 2010). Constatou-se variação na concentração de APs nas espécies do gênero Senecio que causam morte em animais no Brasil. Detectou-se maiores concentrações em S. brasiliensis $(0,31 \%)$, seguido por $S$. heterotrichius $(0,19 \%)$, S. cisplatinus $(0,16 \%)$ e S. selloi $(0,10 \%)$ (Méndez et al. 1990). S. madagascariensis, recentemente identificado no Rio Grande do Sul (Cruz et al. 2009), demonstrou uma concentração de $0,17 \%$ de APs nas partes aéreas (Karam et al. 2009). Os surtos da intoxicação por plantas do gênero Senecio são geralmente de caráter crônico tanto em ruminantes quanto em equinos, semelhante ao observado em bovinos e equinos intoxicados por $C$. retusa. Pequenos ruminantes intoxicados por $C$. retusa, por sua vez, demonstram geralmente quadros de intoxicação aguda. Essa variação é facilmente compreendida, quando levamos em consideração que ovinos e caprinos em áreas infestadas e com escassez de outras forragens ingerem grande quantidade de C. retusa na fase de sementação (maior concentração de APs) e desenvolvem necrose hepática aguda. Nos casos crônicos, os animais ingerem quantidades não suficientes para induzir a forma aguda, mas ocorrem danos hepáticos que serão manifestados posteriormente.

A intoxicação por Senecio spp. é a principal causa de morte em bovinos criados na área de abrangência do LPV/ UFSM, correspondendo a $23 \%$ de todas as causas de morte nessa espécie. A intoxicação por $C$. retusa em equinos no semiárido paraibano foi responsável por aproximadamente $16 \%$ de todas as mortes nessa espécie desde o primeiro diagnóstico no ano de 1993. As mortes causadas por APs em equinos nessa região geralmente são confundidas com raiva ou outras encefalites virais. Nesses casos, a avaliação epidemiológica, os valores de enzimas hepáticas e a patologia são importantes para o diagnóstico preciso dessa toxicose (Pimentel et al. 2009). Os sinais da intoxicação manifestam-se geralmente meses após a ingestão das plantas (Nobre et al. 2004). No Rio Grande do Sul, a ocorrência de intoxicação por Senecio spp. em equinos parece ser muito baixa se comparada com a intoxicação em bovinos, apesar de a espécie ser comprovadamente susceptível a intoxicação experimental por S. brasiliensis (Pilati \& Barros 2007). Uma possível explicação para a baixa ocorrência da intoxicação em equinos seria a menor palatabilidade da planta para esses animais ou a suplementação dos equinos com concentrados no período de menor disponibilidade de pastagens.

Em ovinos a intoxicação por APs foi observada em surtos esporádicos acometendo grande parte dos animais dos rebanhos envolvidos. Os ovinos e caprinos são considerados altamente resistentes à ação dos APs. A resistência é atribuída à maior capacidade de detoxificação pelas enzimas hepáticas, como também a uma maior biotransformação do princípio tóxico pelas bactérias do rúmen (Cheeke 1998). No entanto, podem ocorrer casos de intoxicação em ovinos em áreas muito infestadas por Senecio spp. (llha et al. 2001). Estudos demonstraram que ovinos são altamente susceptíveis à intoxicação aguda por monocrotalina em doses únicas e altas (Nobre et al. 2005, Anjos et al. 2010), porém, essa espécie tem a capacidade de adquirir resistência à intoxicação por $C$. retusa pela administração contínua diária de pequenas doses e não desenvolvem intoxicação quando uma dose tóxica alta é administrada após esse período (Anjos et al. 2009). A resistência adquirida explica a observação de surtos esporádicos de intoxicação por $C$. retusa em pequenos ruminantes no semiárido brasileiro.

Ovinos susceptíveis expostos a doses tóxicas de APs podem desenvolver diferentes quadros clínico-patológicos. A ingestão de doses elevadas de APs durante um curto período de tempo é responsável pela intoxicação aguda associada a necrose hepática centrolobular (Nobre et al. 2005). Uma segunda forma de intoxicação primária por APs, de natureza crônica, está associada ao consumo de doses tóxicas únicas e não suficientes para induzir a doença aguda, mas que induzem fibrose hepática (Anjos et al. 2010). Outros autores afirmam que a doença crônica primária também pode ser induzida pelo consumo prolongado de baixas doses de APs (Bull et al. 1968, Cheeke 1998). Por fim, nesses casos crônicos pode ocorrer a intoxicação crônica secundária hepatógena por cobre, associada ao acúmulo excessivo desse mineral no fígado previamente

Pesq. Vet. Bras. 30(5):447-452, maio 2010 
lesionado (Bull et al. 1968). Os ovinos desenvolvem um quadro agudo de hemólise, icterícia e hemoglobinúria (Ilha et al. 2001).

A intoxicação aguda espontânea por APs em rebanhos brasileiros é relatada em ovinos no semiárido brasileiro na forma de surtos (Nobre et al. 2005). Quando ovinos nãoresistentes são introduzidos em áreas altamente invadidas por $C$. retusa a intoxicação provoca sérias perdas (Nobre et al. 2005). A mortalidade pode variar de $12 \%$ a $57 \%$, com letalidade de $100 \%$. A necrose centrolobular hepática é a lesão característica nos ovinos como também em caprinos. Fibrose hepática e hepatomegalocitose foram lesões observadas em praticamente todos os animais que desenvolveram intoxicação crônica. Os ésteres pirrólicos são agentes alquilantes que inibem a mitose celular, mas a síntese de DNA permanece contínua (Prakash 1999). A soma desses fatores concorre para o aumento do núcleo e do citoplasma (megalocitose) (Stalker \& Hayes 2007). Os metabólicos pirróis também induzem necrose e perda de hepatócitos, que são substituídos por tecido fibroso (Santos et al. 2007).

Lesões hepáticas difusas graves geralmente são acompanhadas de manifestações de encefalopatia hepática (EH). A amônia é considerada a principal substância responsável pela encefalopatia hepática. A consequência do acúmulo de amônia no encéfalo é o edema intramielínico que é morfologicamente denominado degeneração esponjosa ou status spongiosus (Summers et al. 1995). No presente estudo, status spongiosus foi frequentemente observado em ovinos e bovinos na forma crônica da intoxicação por AP. Nos equinos, apesar da EH ter sido observada com maior frequência e intensidade, essa alteração não foi observada. Diferentemente, os equinos com $\mathrm{EH}$ desenvolveram reação astrocitária moderada ou discreta, caracterizada por astrócitos com núcleos tumefeitos e com cromatina vacuolizada e que eram observados isolados, aos pares ou em grupos (astrócitos Alzheimer tipo II) (Summers et al. 1995).

Edemas cavitários, do mesentério e de órgãos digestivos foram observados na maioria dos animais, principalmente na forma crônica da doença. O edema nesses casos é atribuído à hipertensão portal decorrente da fibrose hepática, decréscimo na síntese protéica, ou ambos (Barros et al. 1992). Muitos bovinos e alguns ovinos necropsiados tinham histórico de diarreia intermitente, acompanhada de tenesmo e prolapso de reto. Esse quadro está diretamente relacionado a animais com ascite e edema de mesentério. Assim, o quadro de diarreia pode ser explicado pela compressão do trato alimentar pelo edema.

As lesões mais características da intoxicação por APs no presente estudo foram observadas no fígado (fibrose na forma crônica e necrose centrolobular difusa na forma aguda). Em um surto de intoxicação por $C$. juncea em equinos em Minas Gerais foram relatadas lesões envolvendo principalmente os pulmões, caracterizadas por alveolite fibrosante difusa com espessamente de septos alveolares e infiltrado inflamatório intersticial constituído por macrófagos (Nobre et al. 1994). Nos equinos intoxicados por $C$. retusa neste estudo, as lesões pulmonares consistiram praticamente de edema.

Em resumo, a intoxicação por APs é uma importante causa de morte em ruminantes e equinos no Brasil. A intoxicação crônica por Senecio spp. é a principal causa de morte em bovinos no Rio Grande do Sul e a intoxicação crônica por $C$. retusa é uma das principais causas de morte em equinos no semiárido brasileiro. Ovinos e caprinos são mais resistentes a intoxicação por APs, mas surtos de intoxicação crônica em ovinos por Senecio spp. são observados ocasionalmente no Sul do Brasil. C. retusa é responsável por surtos de intoxicação crônica em ovinos e aguda em ovinos e caprinos em áreas infestadas pela planta no semiárido nordestino.

\section{REFERÊNCIAS}

Anjos B.L., Nobre V.M.T., Dantas A.F.M., Medeiros R.M.T., Oliveira Neto T.S., Molyneux R.J. \& Riet-Correa F. 2010. Poisoning of sheep by seeds of Crotalaria retusa: Acquired resistance by continuous administration of low doses. Toxicon. 55:28-32.

Barros C.S.L., Metzdorf L.L. \& Peixoto P.V. 1987. Ocorrência de surtos de intoxicação por Senecio spp. (Compositae) em bovinos no Rio Grande do Sul. Pesq. Vet. Bras. 7:101-107.

Barros C.S.L., Driemeier D., Pilati C. \& Barros S.S. 1992. Senecio spp. poisoning in cattle in southern Brazil. Vet. Human Toxicol. 34:241246.

Carvalho G.S.T. \& Maugé G.C. 1946. Ação tóxica de Senecio brasiliensis Lessing Fam. Compositae. Revta Fac. Med. São Paulo 3:131136.

Cheeke P.R. 1994. A review of the functional and evolutionary roles of the liver in the detoxification of poisonous plants, with special reference to pyrrolizidine alkaloids. Vet. Human Toxicol. 36:240-247.

Corrêa A.M.R., Bezerra Júnior P.S., Pavarini S.P., Santos A.S., Zlotowisk P., Gomes G. \& Driemeier D. 2008. Senecio brasiliensis (Asteraceae) poisoning in Murrah buffaloes in Rio Grande do Sul. Pesq. Vet. Bras. 28:187-189.

Cruz C.E.F., Karam F.C., Dalto A.C., Pavarini S.P., Bandarra P.M. \& Driemeier D. 2010. Fireweed (Senecio madagascariensis) poisoning in cattle. Pesq. Vet. Bras. 30:10-12.

Culvenor C.C.J. 1980. Alkaloids and human disease, p.124-141. In: Smith R.L. \& Bababunmi E.A. (Eds), Toxicology in the Tropics. Taylor and Francis Ltd, London. 280p.

Curial O. \& Guimarães J.P. 1958. Cirrose hepática enzoótica no cavalo. Mem. Inst. Oswaldo Cruz 56:635-653.

Dantas A.F.M., Nobre V.M.T., Riet-Correa F., Tabosa I.M., Júnior G.S., Medeiros J.M., Silva R.M.N., Silva E.M.N., Anjos B.L. \& Medeiros J.K.D. 2004. Intoxicação crônica espontânea por Crotalaria retusa (Fabaceae) em ovinos na região do semi-árido paraibano, Brasil. Pesq. Vet. Bras. 24(Supl.):18-19.

Gava A. \& Barros C. S. L. 1997. Senecio spp. poisoning of horses in southern Brazil. Pesq. Vet. Bras. 17:36-40.

Ilha M.R.S., Loretti A.P., Barros S.S. \& Barros C.S.L. 2001. Intoxicação espontânea por Senecio brasiliensis (Asteraceae) em ovinos no Rio Grande do Sul. Pesq. Vet. Bras. 21:123-138.

Karam F.S.C., Haraguchi M. \& Gardner D. 2009. Seasonal variation in pyrrolizidine alkaloid concentration and plant development in Senecio madagascariensis Poir. (Asteraceae) in Brazil. $8^{\text {th }}$ International Symposium on Poisonous Plants, João Pessoa, PB, p.89. (Resumo)

Lemos R.A.A. \& Barros C.S.L. 1998. Intoxicação por Crotalaria sp., 
p.322-325. In: Lemos R.A.A. (Ed.), Principais Enfermidade de Bovinos de Corte do Mato Grosso do Sul. Universidade Federal de Mato Grosso do Sul, Campo Grande.

McLean E.K. 1970. The toxic action of pyrrolizidine (Senecio) alkaloids. Pharmacol. Rev. 22:429-483.

Méndez M.C., Riet-Correa F., Schild A.L. \& Garcia J.T.C. 1985. Intoxicação por Echium plantagineum (Boraginaceae) em bovinos no Rio Grande do Sul. Pesq. Vet. Bras. 5:57-64.

Méndez M.C., Riet-Correa F. \& Schild A.L. 1987. Intoxicação por Senecio spp. em bovinos no Rio Grande do Sul. Pesq. Vet. Bras. 7:51-56.

Méndez M.C., Riet-Correa F., Schild A.L. \& Martz W. 1990. Intoxicação experimental por cinco espécies de Senecio em bovinos e aves. Pesq. Vet. Bras. 10:63-69.

Méndez M.C. \& Riet-Correa F. 1993. Intoxication by Senecio tweediei in cattle in southern Brazil. Vet. Human Toxicol. 35:55.

Nobre D., Dagli M.L.Z. \& Haraguchi M. 1994. Crotalaria juncea intoxication in Horses. Vet. Human. Toxicol. 36:445-448.

Nobre V.M.T., Dantas A.F.M., Riet-Correa F., Barbosa Filho J.M., Tabosa I.M. \& Vasconcelos J.S. 2005. Acute intoxication by Crotalaria retusa in sheep. Toxicon 45:347-352.

Nobre V.M.T., Riet-Correa F., Dantas A.F.M., Tabosa I.M., Medeiros R.M.T. \& Barbosa Filho J.M. 2004a. Intoxication by Crotalaria retusa in ruminats and equidae in the state of Paraíba, Northeaster Brazil, p.275-279. In: Acamovich T., Stewart C.S., Pennycott T.W. (Eds), Plants Poisoning and Related Toxins. CAB International, Glasgow, UK.
Nobre V.M.T., Riet-Correa F., Barbosa Filho J.M., Tabosa I.M. \& Vasconcelos J.S. 2004b. Intoxicação por Crotalaria retusa (Fabaceae) em eqüinos no semi-árido da Paraíba. Pesq. Vet. Bras. 24:132-143.

Pilati C. \& Barros C.S.L. 2007. Intoxicação experimental por Senecio brasiliensis (Asteraceae) em eqüinos. Pesq. Vet. Bras. 27:287-296.

Pimentel L.A., Oliveira D.M., Galiza G.J.N., Rego R.O., Dantas A.F.M. \& Riet-Correa F. 2009. Doenças do sistema nervoso central de equídeos no semi-árido. Pesq. Vet. Bras. 29:589-597.

Prakash A.S., Perera T.N., Reilly P.E.B. \& Seawright A.A. 1999. Pirrolizidine alkaloids in human diet. Mutat. Res. 443:53-67.

Riet-Correa F. \& Medeiros R.M.T. 2001. Intoxicações por plantas em ruminantes no Brasil e no Uruguai: importância econômica, controle e riscos para a saúde pública. Pesq. Vet. Bras. 21:38-42.

Rissi D.R., Rech R.R., Pierezan F., Gabriel A.L., Trost M.E., Brum J.S., Kommers G.D. \& Barros C.S.L. 2007. Intoxicações por plantas e micotoxinas associadas a plantas em bovinos no Rio Grande do Sul: 461 casos. Pesq. Vet. Bras. 27:261-268.

Stalker M.J. \& Hayes M.A. 2007. Liver and biliary system, p.297-388. In: Maxie M.G. (Ed.), Jubb, Kennedy and Palmer's Pathology of Domestic Animals. Vol.2. $5^{\text {th }}$ ed. Saunders Elsevier, Philadelphia.

Santos J.C.A., Riet-Correa F., Simões S.V.D. \& Barros C.S.L. 2008. Patogênese, sinais clínicos e patologia das doenças causadas por plantas hepatotóxicas em ruminantes e eqüinos no Brasil. Pesq. Vet. Bras. 28:1-14.

Summers B.A., Cummings J.F. \& de Lahunta A. 1995. Degenerative diseases of the central nervous system, p.208-214. In: Ibid. (Eds), Veterinary Neuropathology. Mosby, St Louis. 525p. 